doi:10.17659/01.2020.0034

Journal of Case Reports 2020;10(2):127-131

\title{
Fatal Toxic Megacolon during Exacerbation of Ulcerative Colitis in Puerperium
}

\author{
Pankaj S Ghormade, Bhoj Kumar Sahu, Bedanta Sharma \\ Department of Forensic Medicine and Toxicology, All India Institute of Medical Sciences, Raipur, Chhattisgarh, India-492099.
}

\section{Corresponding Author:}

Dr. Pankaj S Ghormade

Email: pankajforensic@gmail.com

This is an Open Access article distributed under the terms of the Creative Commons Attribution License (creativecommons.org/ licenses/by/3.0).

Received : January 27, 2020

Accepted : June 14, 2020

Published : June 25, 2020

\begin{abstract}
Background: Ulcerative colitis (UC) is an inflammatory bowel disease (IBD), which affects mainly the young adults of either sex. It has been estimated that between 30 to $50 \%$ of the female patients with $\mathrm{UC}$ will have an exacerbation during pregnancy or in the early postpartum period. A rare but potentially fatal complication of UC is toxic megacolon. Case Report: We describe the case of a 22-year-old multiparous married woman in the puerperal period, she had been suffering from a chronic gastro-intestinal problem for two years, which was exacerbated after delivery. Her clinical condition remains undiagnosed despite prominent signs and symptoms with laboratory investigations suggestive of complication of inflammatory bowel disease (IBD). Conclusion: Gross findings on autopsy and histopathology ruled out other differential diagnoses for toxic megacolon due to UC. This rare case highlights the exacerbation of UC in postpartum (puerperal) period. Autopsy findings in such cases are crucial in deciding allegations of medical negligence.
\end{abstract}

Keywords: Gastrointestinal Diseases, Inflammatory Bowel Diseases, Postpartum Period, Pregnancy, Toxic Megacolon.

\section{Introduction}

Ulcerative colitis (UC) is a type of inflammatory bowel disease (IBD) that mainly affects young adults of either sex. The incidence of ulcerative colitis is 10 to 12 cases per 100,000 people and the prevalence rate is 35 to 100 cases per 100,000 people [1]. UC leading to toxic megacolon represents an extreme in the spectrum of severe colitis [2] and complicates the presentation in 5\% of patients admitted with severe acute colitis [3]. It is formally defined as the total or segmental nonobstructive dilatation of the colon associated with systemic symptoms $[4,5]$. Other causes for toxic megacolon include Crohn's disease, ischemic colitis, Clostridium difficile colitis, HIV infection, cytomegalovirus infection, drug-induced colitis [6]. The development of toxic colitis in pre-existing $\mathrm{UC}$ is uncommon during pregnancy and usually associated with an unfavorable outcome [7].
The management of toxic megacolon involves the treatment of the underlying cause, attenuation of colitis, treatment of toxemia, and circumvention of further complications, specifically bowel perforation. Close medical monitoring and supportive care are imperative [8]. The mortality is increased in colonic perforation, peritonitis, septic shock and multiple organ dysfunction, so its early diagnosis and treatment are crucial [9]. Herewith, we dealt with a rare fatal case of toxic megacolon associated with UC exacerbated in the puerperal period.

\section{Case Report}

A 22-year-old married woman, of low socioeconomic background, was submitted to autopsy because of the unknown cause of death. On reviewing treatment records and history from her husband, she was multiparous and delivered a female child by vaginal delivery at a local 
community health center one and a half months ago. The course of her pregnancy was uneventful. In the puerperal period, she started complaining of nausea, vomiting, constipation, bloody stools, flatulence, decreased appetite, intermittent fever and infrequent abdomen pain relieved after defecation for which she was taking treatment from a local general practitioner. She was prescribed, a pain killer along with antacids, apparently based on her symptoms. Her condition started worsening despite taking the prescribed medication nearly for one month. In sequence of events, she was admitted to nearby multi-specialty private hospital. Patient had high grade fever, tachycardia, tachypnea and subsequently developed hypotension, decreased urine output and altered mental status. Laboratory workup revealed: hemoglobin of $5.4 \mathrm{~g} \%$ (reference range [RR]: 11-14), leukocytes of $20600 / \mathrm{mm}^{3}$ (RR: 4500-11000) with neutrophilia, platelets of $1,50,000 / \mathrm{mm}^{3}$ (RR: 150000-450000), total bilirubin of $2.5 \mathrm{mg} / \mathrm{dL}$ (RR: 0.1-1.2); total protein $3.85 \mathrm{mg} / \mathrm{dL}$ (RR: 5.5-8.6), urea $57 \mathrm{mg} / \mathrm{dL}$ (RR: 15 45), creatinine: $1.4 \mathrm{mg} / \mathrm{dL}$ (RR: 0.6-1.2), potassium $2 \mathrm{mEq} / \mathrm{L}$ (3.6-5.2); sodium $120 \mathrm{mEq} / \mathrm{L}$ (135 - 145); C-reactive protein $16 \mathrm{mg} / \mathrm{L}$ (RR 0-10), erythrocyte sedimentation rate $64 \mathrm{~mm} / \mathrm{hr}(\mathrm{RR}:<20)$, hepatitis $\mathrm{B}, \mathrm{C}$ and HIV non-reactive. Ultrasonography (USG) revealed free fluid in peritoneum and

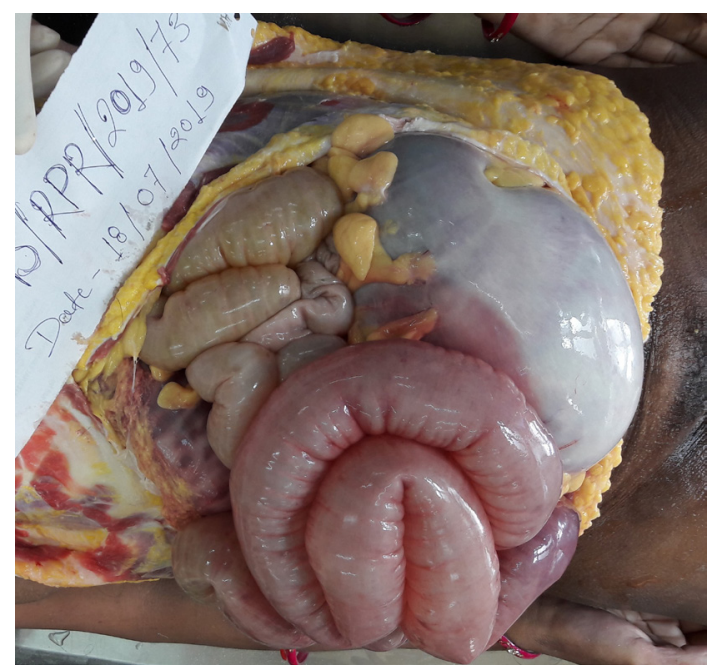

Fig.1: Gross distention of sigmoid colon and descending colon after opening of the abdominal cavity. gaseous distention. Treating physician made a provisional diagnosis of acute febrile illness with sepsis with acute renal failure and severe anemia with electrolyte disturbance. Despite treatment, her condition deteriorated significantly with constant diffuse abdominal pain, abdominal distension and increased frequency of bloody diarrhea. The patient was referred to our tertiary care institute for further management, wherein she was declared brought dead. As the case remained clinically undiagnosed autopsy was conducted. The patient had a history of symptoms of abdominal pain, constipation, flatulence, and intermittent bloody stool for two years.

The deceased was a thin built with BMI of 17.8. On external examination, there was generalized pallor. Signs of recent pregnancy were present. The abdomen was distended. On internal examination all the organs were pale without significant gross pathological findings except in intestines and spleen. The intestinal coils were distended with massive distention of descending colon and sigmoid colon without obstruction or volvulus [Fig.1]. The peritoneal cavity contained about $250 \mathrm{ml}$ of turbid yellowish colored free fluid. The stomach was empty with pale mucosa. The spleen was soft, flabby, enlarged, weighing 210 g (RR:131-164 gms). Greenish-yellow colored, foul-smelling contents and gases were present in jejunum and ileum. On dissection, about 1 liter of dark red fluid blood was present due to continuous luminal bleeding of the descending and sigmoid colon with foul smell [Fig.2]. There was continuous and diffuse involvement of the rectum back into the sigmoid colon and the descending colon. Flattened hemorrhagic mucosa with extensive linear ulcers

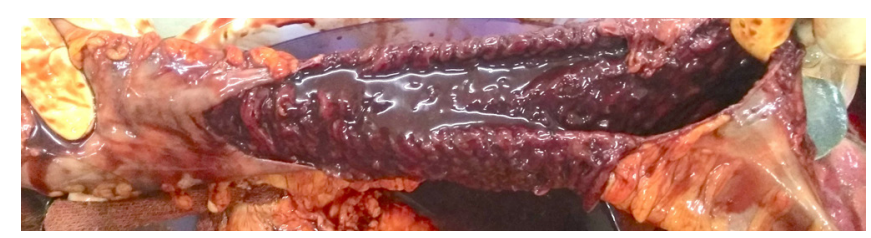

Fig.2: Macroscopic view of the bleeding into lumen of descending colon. 
(blue arrow) and psuedopolyp's (curved arrow) along axis of involved bowel was found. Diffuse mucosal inflammation and necrotic changes were also noted in the sigmoid colon [Fig.3]. We did not find deep fissuring ulceration, no strictures or fistulas, no sinus tract formation, no small intestinal involvement, no bowel wall thickening. Uterus measured $11 \times 7 \times 3 \mathrm{~cm}$ and weighed $140 \mathrm{gms}$ (RR: 65 to 96 gms).

Histopathology revealed neutrophilic infiltration into the mucosal and sub-mucosal layer of the affected large intestine suggestive of cryptitis with distortion of the crypt architecture [Fig.4]. We also found significant histological findings of sepsis in other organs that included bilateral patchy consolidation, splenitis and acute tubular necrosis. We ruled out Crohn's disease as it commonly involves terminal ileum and/or ascending colon with segmental involvement of bowel with skip areas and serpiginous ulcers, which may develop into fissures. Microscopically, it shows transmural non-caseating granulomas and fibrosis. Splenic flexure is primarily affected in ischemic colitis and external surface of affected bowel has fusiform or saccular dilatation. Microscopically, ulcerated areas show sub-mucosal thickening due to fibrosis in ischemic colitis. Infectious enterocolitis due to Clostridium difficile, Shigella etc. causes formation of pseudo-membrane composed of network of fibrin and mucus, in which are entangled inflammatory cells and mucosal epithelial cells. The inflammation spreads laterally rather than deeply and the lesions may be confined, to the large intestine or small intestine, or both may be involved. The mucosa of the bowel is covered by patchy, raised yellowwhite plaques in pseudomembranous enterocolitis [10].

Gross and histological findings in our case also excludes possibility of Ogilvie's syndrome (non-obstructive colonic) caused due to parasympathetic dysfunction [11]. Hence, we have concluded cause of death as shock and toxemia due to toxic megacolon with ulcerative colitis based

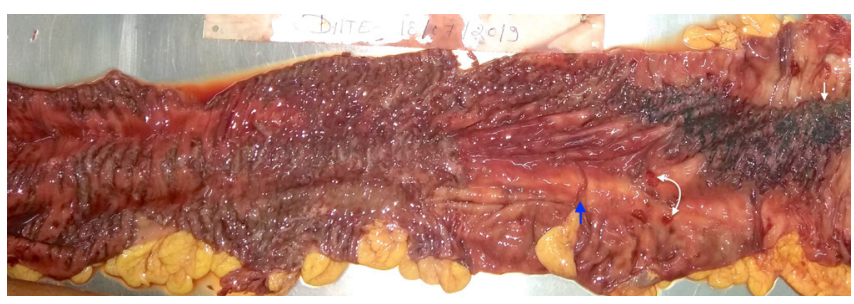

Fig.3: Macroscopic view of the flattened hemorrhagic mucosa with extensive linear ulcers (blue arrow), pseudopolyps (curved arrow) and necrotic changes in sigmoid colon (white arrow).

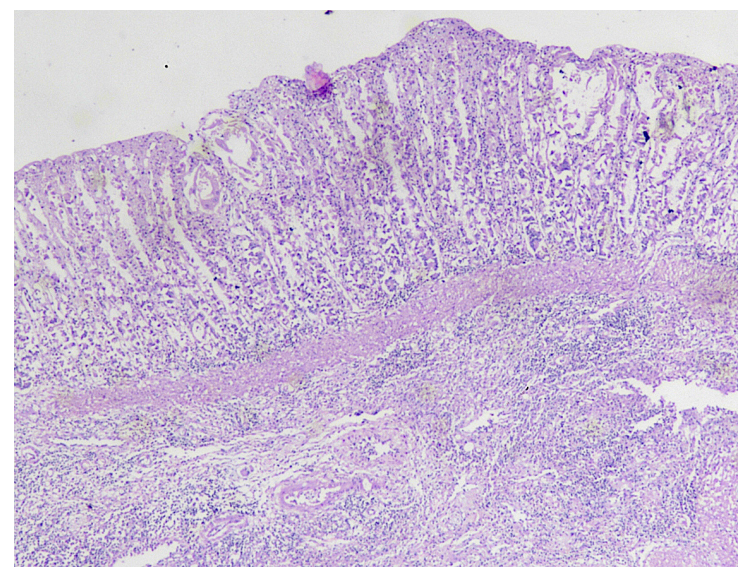

Fig.4: Colon depicting infiltration of mononuclear inflammatory cells into the mucosal and submucosal layer of the affected large intestine suggestive of cryptitis with ulceration of mucosa due to UC (H and E 10X).

upon gross and histological finding, available laboratory investigations report and clinical records of the patient.

\section{Discussion}

The progression of $\mathrm{UC}$ in the antenatal period correlates with the onset of pregnancy. If it is in the remission phase at the time of conception, it will remain quiescent throughout pregnancy, while if it is active, it will progressively worse, leading to an increased incidence of stillbirth, preterm delivery, or post-partum complications for both mother and neonate [12]. It has been estimated that between 30 to $50 \%$ of female patients with pre-existing UC will have an exacerbation during pregnancy or in the early post-partum period [13]. Whereas 
patients presenting for the first time with ulcerative colitis, only a minority (5-8\%) manifest symptoms and signs of severe acute colitis and are at the highest risk of developing toxic megacolon at an early stage of the disease [4].

In our case, the outcome of pregnancy was uneventful; however, severe colitis developed in puerperal period and a risk factor for such factor remains undetermined. The hallmark of clinical diagnostic criteria of toxic megacolon as suggested by Jalan et al.[14] are (i) radiographic evidence of colonic dilatation (more than $6 \mathrm{~cm}$ in transverse colon), (ii) any 3 of these (fever $>101.5^{\circ} \mathrm{F}$, tachycardia $>120$ beats/min, leucocytosis $>10.5 \mathrm{x}$ $10^{3} / \mu \mathrm{L}$, anemia), (iii) any one of these (dehydration, altered mental status, electrolyte abnormality, hypotension). Similarly, in our case; we noticed presence of all the clinical diagnostic criterion of toxic megacolon except non-specific USG finding as described above. Plain X-ray imaging, CT abdomen and colonoscopy was not advised by the treating physician, hence, not performed in this particular case.

We found continuous involvement of rectum back into the sigmoid colon and descending colon with specific gross and histological findings consistent with UC as reported in the literature [10]. Hence, massive non-obstructive dilatation of involved bowel loops is indicative of toxic mega-colon due to complication of UC. We rule out other differential causes of toxic megacolon such as Crohn's disease, ischemic colitis, infective pseudomembranous enterocolitis, Ogilvie syndrome etc. as described above. Diagnosis of $\mathrm{UC}$ is made by clinical evaluation for systemic manifestations and various imaging studies depicting the dilatation of colon. Plain abdominal imaging (X-ray erect abdomen) is still the most established radiological tool for detecting colonic dilatation. However, confirmatory diagnosis is made by finding of histology of colonoscopy biopsy $[4,15]$.
However, in our case, the treating physician failed to elicit proper history and interpret clinical presentation of the case suggestive of complications like IBD and also overlooked laboratory investigation reports, hence diagnostic radiological imaging was not advised. It led to misdiagnosis and mismanagement of the case. Antacids were given merely in anticipation of the presence of gastroesophageal reflux disease. Such type of missed diagnosis is not uncommon in clinical practice. Hence, considerable time was lost in referring the case to the tertiary care institute. Due to misdiagnosis patient received non-specific treatment. However, clinical signs and symptoms in our case and findings of laboratory investigations were consistent with diagnostic criterion of toxic megacolon [14].

\section{Conclusion}

Hence, this rare case discusses the differential diagnoses of toxic megacolon due to UC on autopsy. In clinically undiagnosed cases with an allegation of medical negligence, the forensic pathologist can help to conclude the cause of death by meticulous autopsy.

Contributors: PSG: manuscript writing and the conception of the work; BKS, BS were responsible for revising it critically. PSG will act as a study guarantor. All authors approved the final version of this manuscript and are responsible for all aspects of this study.

Funding: None; Competing interests: None stated.

\section{References}

1. Strong SA. Management of acute colitis and toxic megacolon. Clin Colon Rectal Surg. 2010;23:274-284.

2. Marshak RH, Lester LJ. Megacolon a complication of ulcerative colitis. Gastroenterol.1950;16:768-772.

3. Gan SI, Beck PL. A new look at toxic megacolon: an update and review of incidence, etiology, pathogenesis, and management. Am J Gastroenterol. 2003;98:23632371.

4. Sheth SG, LaMont JT. Toxic megacolon. Lancet. 1998;351:509-513.

5. Jones JH, Chapman M. Definition of megacolon in colitis. Gut. 1969;10:562-564. 
6. Shivashankar R, Khanna S, Kammer PP, Harmsen WS, Zinsmeister AR, Baddour LM, et al. Clinical factors associated with development of severe-complicated Clostridium difficile infection. Clin Gastroenterol Hepatol. 2013;11:1466-1471.

7. Korelitz BI. Inflammatory bowel disease and pregnancy. Gastroenterol Clin North Am. 1998;27:213-224.

8. Caprilli R, Vernia P, Latella G, Torsoli A. Early recognition of toxic megacolon. J Clin Gastroenterol. 1987;9:136-142.

9. Farmer RG, Easley KA, Rankin GB. Clinical patterns in ulcerative colitis: disease location, progression and prognosis. Dig Dis Sci. 1993;38:1137-1146.

10. Mohan H. Textbook of Pathology: $6^{\text {th }}$ Edition; Delhi: Jaypee Brothers Medical Publishers Ltd. 2010: pp. 565568.
11. Maloney N, Vargas HD. Acute intestinal pseudoobstruction (Ogilvie's syndrome). Clin Colon Rectal Surg. 2005;18:96-101.

12. Boulton R, Hamilton M, Lewis A, Walker P, Pounder R. Fulminant ulcerative colitis in pregnancy. Am J Gastroenterol. 1994;89:931-933.

13. Anderson JB, Turner GM, Williamson RCN. Fulminant $\mathrm{UC}$ in late pregnancy and the peurperium. JRSM. 1987;80:492-494.

14. Jalan KN, Sircus W, Card WI, Falconer CW, Bruce CB, Crean GP, et al. An experience of ulcerative colitis I. Toxic dilation in 55 cases. Gastroenterol. 1969;57:68-82.

15. Caprilli R, Vernia P, Latella G, Torsoli A. Early recognition of toxic megacolon. J Clin Gastroentero. 1987; 9:136-142. 